\title{
Dysregulated genes and their functional pathways in luteinized granulosa cells from PCOS patients after cabergoline treatment
}

\author{
H Ferrero ${ }^{1,2, *}$, P Díaz-Gimeno ${ }^{1,2, *}$, P Sebastián-León ${ }^{1,2}$, A Faus ${ }^{1}$, R Gómez ${ }^{2}$ and A Pellicer ${ }^{1,3}$ \\ ${ }^{1}$ Fundación IVI, Instituto Universitario IVI, Universidad de Valencia, Valencia, Spain, ${ }^{2}$ Instituto de Investigación \\ Sanitaria INCLIVA, Valencia, Spain and ${ }^{3}$ Instituto de Investigación Sanitaria Hospital Universitario y Politécnico La \\ Fe, Valencia, Spain
}

Correspondence should be addressed to R Gómez; Email: raulgomgal@gmail.com

* $(\mathrm{H}$ Ferrero and P Díaz-Gimeno contributed equally to this work)

\begin{abstract}
Polycystic ovarian syndrome (PCOS) is a common reproductive disorder frequently associated with a substantial risk factor for ovarian hyperstimulation syndrome (OHSS). Dopamine receptor 2 (D2) agonists, like cabergoline (Cb2), have been used to reduce the OHSS risk. However, lutein granulosa cells (LGCs) from PCOS patients treated with Cb2 still show a deregulated dopaminergic tone (decreased D2 expression and low dopamine production) and increased vascularization compared to non-PCOS LGCs.

Therefore, to understand the PCOS ovarian physiology, it is important to explore the mechanisms that underlie syndrome based on the therapeutic effects of $\mathrm{Cb} 2$. Here, LGCs from non-PCOS and PCOS patients were cultured with hCG in the absence/presence of $\mathrm{Cb} 2(n=12)$. Subsequently, a transcriptomic-paired design that compared untreated vs treated LGCs within each patient was performed. After transcriptomic analysis, functions and genes were prioritized by systems biology approaches and validated by RT-qPCR. We identified that similar functions were altered in both PCOS and non-PCOS LGCs treated with Cb2; however, PCOStreated LGCs exhibited more significant changes than non-PCOS. Among the prioritized functions, dopaminergic synapse, vascular endothelial growth factor (VEGF) signaling, apoptosis and ovarian steroidogenesis were highlighted. Finally, network modeling showed CASP9, VEGFA, AKT1, CREB, AIF, MAOA, MAPK14 and BMAL1 as key genes implicated in these pathways in Cb2 response, which might be potential biomarkers for further studies in PCOS.

Reproduction (2018) 155 373-381
\end{abstract}

\section{Introduction}

Polycystic ovarian syndrome (PCOS) is a common reproductive disorder frequently associated with various metabolic abnormalities and substantially leading to the increased risk for ovarian hyperstimulation syndrome (OHSS) after administration of exogenous gonadotropins. According to the Rotterdam criteria established in 2004 (Rotterdam ESHRE/ASRM-Sponsored PCOS Consensus Workshop Group 2004), women with PCOS are diagnosed by the presence of at least two of the three following criteria: clinical and biochemical signs of hyperandrogenism, anovulation (oligo- or amenorrhea) and/or polycystic ovaries. In general, PCOS is associated with infertility: a lower chance of conceiving and higher miscarriage rates (Pasquali \& Gambineri 2006, Goverde et al. 2009). For this reason, women with PCOS often have to undergo assisted reproduction techniques, which involve conventional treatments to induce ovulation by exogenous gonadotropins and in vitro maturation (Norman \& Clark 1998). However, these treatments may lead to a higher risk of OHSS (Van Wely et al. 2003) in these women: $15 \%$ of women with PCOS undergoing ovulation treatment suffer from severe OHSS, compared to $3 \%$ of control women (Swanton et al. 2010). In this regard, dopamine receptor 2 (D2) agonists (D2-ags) have been successfully used in women with PCOS undergoing ovulation treatment to reduce the risk of OHSS (Papaleo et al. 2001, Guvendag Guven et al. 2013).

Granulosa cells (GCs) are important in ovarian folliculogenesis because they provide a suitable microenvironment for follicular development and oocyte maturation (Lan et al. 2015). Therefore, a recent study performed by our group assessed lutein GCs (LGCs) from women without and with PCOS treated with a D2-ag, cabergoline (Cb2) (the D2-ag most used in clinic treatment), to examine the mechanism of D2 inhibition. We demonstrated that PCOS LGCs present a deregulated dopaminergic tone (decreased D2 expression and low dopamine production) and increased vascularization compared to non-PCOS LGCs (Gómez et al. 2011). PCOS LGCs are known to exhibit alterations in vascular endothelial growth factor (VEGF) signaling (Agrawal et al. 2002), ovarian steroidogenesis (Eisner et al. 2002) and apoptosis (Karuputhula et al. 2013) pathways, 
which could promote increased OHSS risk. Therefore, to understand the PCOS ovarian physiology, it is important to explore the mechanisms that underlie syndrome based on the therapeutic effects of $\mathrm{Cb} 2$.

Microarrays are useful tools to measure the expression of thousands of genes (the transcriptome) within a particular mRNA sample. These expression profiles can be used to understand the transcriptomics of cell behavior in diseases and identify diagnostic biomarkers and personalized responses to reproductive therapies. For these reasons, several studies have focused on describing the GC transcriptome in reproductive diseases. Microarray studies have observed differentially expressed genes in GCs at different stages of folliculogenesis (Douville \& Sirard 2014, Golini et al. 2014), in relation to oocyte quality (Ouandaogo et al. 2011, Al-Edani et al. 2014) and in association with PCOS (Lan et al. 2015). Despite the relevance of Cb2 in the prevention of OHSS risk, to date, no complete study on GCs from women with PCOS treated with $\mathrm{Cb} 2$ has been reported to elucidate the molecular mechanism underlying $\mathrm{Cb} 2$ 's effect. Hence, the main goal of this study was to assess the transcriptomic profiles of LGCs from women with and without PCOS using Affymetrix microarray chips to provide novel information about the molecular changes that occur in these cells when they are treated with a D2-ag (Cb2). We also assessed the signal transduction pathways regulated by this treatment using systems biology as a novel approach in ovarian transcriptomics to better understand such a complex system.

\section{Materials and methods}

\section{Sample collection}

Follicular fluid-derived LGCs were isolated from women without PCOS $(n=3)$ as a control group, and women with PCOS $(n=3)$ as determined by achieving $2 / 3$ of the Rotterdam criteria (Rotterdam ESHRE/ASRM-Sponsored PCOS Consensus Workshop Group 2004). LGCs were obtained at the time of oocyte retrieval from women aged 25 to 32 years, who had normal response profiles to controlled ovarian hyperstimulation $(\mathrm{COH})$ protocols (oocytes retrieved, 7-20; E2 $<189 \mathrm{pg} / \mathrm{mL}$ in follicular phase; body mass index (BMI) $<30$ )
(Table 1). Women gave written informed consent, and the study protocol was approved by the ethics committee at CEICIVI Valencia (1503-VLC-014-AP).

\section{LGC isolation and culture}

LGCs were isolated using our previously described filter method (Ferrero et al. 2012). Isolated LGCs were seeded in 24-well cell culture plates at a density of 50,000 cells/well and incubated overnight $\left(37^{\circ} \mathrm{C}, 5 \% \mathrm{CO}_{2}\right)$ to enable removal of non-adherent cells. Subsequently, LGCs from each patient were washed and cultured in M-199 with 10\% fetal bovine serum (Labclinics, Barcelona, Spain) and $5 \mathrm{IU} / \mathrm{mL}$ hCG (Profasi: Serono Laboratories, Madrid, Spain), in the absence or presence of the D2-ag (cabergoline; Cb2) at $100 \mu \mathrm{M}$ (Dostinex: Pharmacia \& Upjohn, North Peapack, NJ, USA) for $72 \mathrm{~h}\left(37^{\circ} \mathrm{C}, 5 \% \mathrm{CO}_{2}\right)$. Cabergoline doses and administration days and doses of hCG were established in our previous study, demonstrating that LGCs are sensitive to hCG in the first culture days (Ferrero et al. 2014b).

Thus, this study included four different analysis groups: $\mathrm{Cu}$ : control untreated (non-PCOS, untreated $(n=3)$ ), Ct: control treated (non-PCOS, treated $(n=3))$, Pu: PCOS untreated $(n=3)$ and Pt: PCOS treated $(n=3)$.

\section{LGC collection, RNA extraction and microarray hybridization}

After treatment, total mRNA from the cells was extracted using the Quick-RNA Microprep kit (Zymo Research, Freiburg, Germany), according to the manufacturer's instructions. Quality and concentration of the mRNA samples were determined using the RNA Nano chip (Agilent Technologies) and analyzed by the Agilent 2100 Bioanalyzer (Agilent Technologies). mRNA extracts were stored at $-80^{\circ} \mathrm{C}$ until further use for array analysis. Microarray experiments were conducted according to the manufacturer's instructions (Central Research UnitINCLIVA; University of Valencia, Spain). RNA was hybridized to Human 1.0 ST GeneChip Arrays (Affymetrix). The array includes $>750,000$ unique 25-mer oligonucleotide transcripts, thus constituting $>28,000$ well-annotated genes. Analyses were performed by using one patient sample per GeneChip. Files were captured using an Affymetrix GeneChip Scanner 3000 7G. To verify if microarray results were reliable and sample quality was good, RNA samples from four of the 12 conditions were randomly selected to be replicated as a technical control.

Table 1 Characteristics of individual non-PCOS and PCOS patients.

\begin{tabular}{|c|c|c|c|c|c|c|c|c|}
\hline & \multirow[b]{2}{*}{ Age } & \multirow[b]{2}{*}{ BMI } & \multirow[b]{2}{*}{ Oocytes } & \multicolumn{2}{|c|}{ Follicular Phase } & \multirow{2}{*}{$\begin{array}{c}\text { PRL 1.39-24.2 } \\
\quad(\mathrm{ng} / \mathrm{mL})\end{array}$} & \multirow[b]{2}{*}{ Menstrual cycle } & \multirow[b]{2}{*}{ Ovaries } \\
\hline & & & & E2: $\mathbf{3 9 - 1 8 9}(\mathrm{pg} / \mathrm{mL})$ & LH: 1-18 (IU/mL) & & & \\
\hline Non-PCOS 1 & 25 & 22.76 & 7 & 29 & 6.25 & 5.5 & Eumenorrhea & Normal \\
\hline Non-PCOS 2 & 30 & 22.58 & 17 & 45.65 & 11.6 & 10.26 & Eumenorrhea & Normal \\
\hline Non-PCOS 3 & 27 & 20.78 & 15 & 80.21 & 1.6 & 3.5 & Eumenorrhea & Normal \\
\hline PCOS 1 & 23 & 19.48 & 20 & 39 & $80.89 *$ & $51.02 *$ & Amenorrhea* & Polycystic ovaries* \\
\hline PCOS 2 & 32 & 24.12 & 5 & 49.8 & 1.7 & 16.85 & Oligomenorrhea* & Polycystic ovaries* \\
\hline PCOS 3 & 29 & 23.57 & 9 & 36 & $6.96^{*}$ & 11.19 & Amenorrhea* & Polycystic ovaries* \\
\hline
\end{tabular}

* Meets with Rotterdam criteria.

$\mathrm{BMI}$, body mass index; E2, estradiol; LH, luteinizing hormone; PRL, prolactin. 


\section{Microarray preprocessing and normalization}

Analyses were performed using the $\mathrm{R}$ programing language, version 3.2.0 (R Core Team 2013). Affymetrix raw data (CEL files) was preprocessed by background correction, logarithmic transformation and normalization. Background correction was assessed using the robust multi-array average (RMA) method (Irizarry et al. 2003) and microarray intensity normalization was performed using the quantile method (Bolstad et al. 2003). Box plot diagrams were analyzed for detecting outliers. Microarray data are available at Gene Expression Omnibus (GSE98595).

\section{Exploratory and differential expression analysis}

Principal component analysis (PCA) was used as an exploratory method to detect sample behavior and the effects of other uncontrolled variables, using the Prcomp function from R, version 3.2. Differential gene expression assessment of all comparisons was carried out using Limma moderated t-statistics (Ritchie et al. 2015). Standard microarray analysis techniques for comparisons were carried out using one test for each gene (or probe-set) in the microarray. To minimize false positives in the study, raw $P$ values were corrected for multiple testing to derive adjusted $P$ values (false discovery rate (FDR)) (Benjamini \& Hochberg 1995).

\section{Functional profiling}

Gene Set Enrichment Analysis (GSEA) was carried out for each of the comparisons explored in the study. To discover functional blocks enriched in the conditions, we used logistic regression models (Sartor et al. 2009, Montaner \& Dopazo 2010). The conventional multiple testing $P$ value correction procedure proposed by Benjamini and Hochberg (1995) was used to derive adjusted $P$ values. Functional blocks from the Gene Ontology Biological Process (Ashburner et al. 2000) (GO BP) and Kyoto Encyclopedia of Genes and Genomes (KEGG) databases (Kanehisa et al. 2012) were used in this study. Ovarian-specific databases were consulted to contextualize our results (Hsueh \& Rauch 2012, Mary et al. 2016). To analyze the main regulator genes between the prioritized functions, a network analysis was implemented using Cytoscape (Cline et al. 2007). Network modeling allowed us to identify main genes related to their position in the network, based on network medicine principles, in which genes that connect various functions are highlighted as key regulators (Barabási et al. 2011).

\section{Quantitative fluorescent real-time PCR}

To corroborate the gene expression obtained in the array analysis, $300 \mathrm{ng}$ of mRNA from the same samples used in the microarray (Cu and $\mathrm{Ct}$; $\mathrm{Pu}$ and $\mathrm{Pt}$ ) were reverse-transcribed into cDNA using the PrimeScript RT reagent kit (Takara Bio) and quantified by Qubit. CDNA expression levels of the genes caspase 9 (CASP9), VEGFA, RAC-alpha serine/threonine protein kinase $(A K T 1)$, apoptosis-inducing factor $(A I F)$, monoamine oxidase A $(M A O A)$, mitogen-activated protein kinase 14 (MAPK14) and brain and muscle Arnt-like protein-1 (BMAL1) were estimated by quantitative real-time PCR (RT-qPCR) using the Power-Up SYBR Green master mix (Applied Biosystems). Expression levels were normalized to the house keeping gene $\beta$-actin $(A C T B)$ quantified by the $\Delta \Delta C t$ method and expressed as the fold change in each group.

\section{Statistical analysis}

Statistical analysis comparing both RNA expression methodologies was performed using $R$ statistical computing $(R$ Core Team 2013) and data were expressed as fold changes. To compare treated and untreated groups in each condition (non-PCOS and PCOS), a paired t-test comparison was used to analyze the expression of CASP9, VEGFA, AKT1, $M A O A$ and BMAL1, which were normally distributed. The non-parametric paired-samples Wilcoxon test was used to analyze the expression of AIF and MAPK14 that were not normally distributed.

\section{Results}

\section{Transcriptomic analysis}

Full human transcriptome analysis revealed a clustering effect in the LGCs treated with or without Cb2. Equally, the transcriptomes in LGCs from non-PCOS and PCOS were grouped depending on the presence/absence of the syndrome, showing a gene expression response (Fig. 1A) and indicating that there were baseline differences between groups.

Subsequently, microarray data revealed 137 genes differentially expressed between Ct vs Cu and 290 genes between Pt vs Pu (Fig. 1B) (FDR <0.05), demonstrating that $\mathrm{Cb} 2$ influenced the expression of more genes in PCOS than non-PCOS (control) samples. Notably, only $10.07 \%$ (43 genes) of the differentially expressed genes were shared in both treated vs untreated comparisons (Fig. 1C), showing the different effect of Cb2 in PCOs. In addition, analysis in Pu vs $\mathrm{Cu}$ showed that most of the differentially expressed genes at baseline in untreated PCOS vs untreated controls were independent of dysregulated genes in the $\mathrm{Cb} 2$ treatment, corroborating that the baseline differences due to PCOS did not affect our results (Supplementary Fig. 1A, see section on supplementary data given at the end of this article).

\section{Functional dysregulation in Cb-2-treated PCOS cells}

The main functions affected after $\mathrm{Cb} 2$ treatment are shown in Fig. 2. There were $388 \mathrm{GO}$ terms enriched from the Ct vs Cu comparison and 661 from the Pt vs $\mathrm{Pu}$ comparison. For the functional interpretation of all these enriched GO terms (FDR <0.05), 17 functions were assigned manually to group and summarize all the terms: Vesicles transport; Response, transduction and signaling; Reproductive related process; Ossification; Neurosystem; Lipid metabolism; Immune system; homeostasis and transport; Energetic and respiratory 

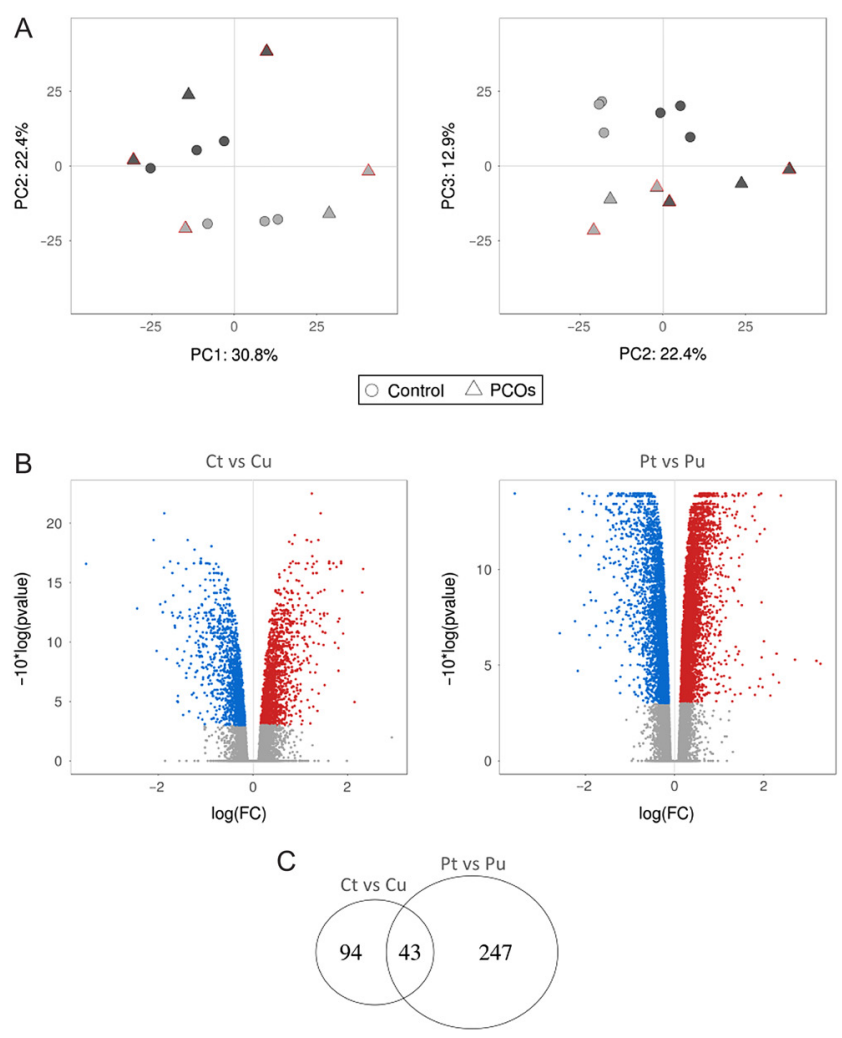

Figure 1 Transcriptomic analysis. (A) Principal component analysis (PCA) of full transcriptomes in LGCs from women without (nonPCOS) and with PCOS treated and untreated with D2-ag (Cb2). Ct: non-PCOS (control) treated ( $n=3)$; Cu: non-PCOS (control) untreated $(n=3) ;$ Pt: PCOS treated $(n=3) ;$ Pu: PCOS untreated $(n=3)$. PCA Component 1 (PC1)/Component 2 (PC2) revealed a clustering effect related to treatment with (dark shapes) or without D2-ag (Cb2) (bright shapes) (top left), and PCA Component 2 (PC2)/Component 3 (PC3) clustered the samples related to the disease (triangles $=$ PCOS; circles $=$ non-PCOS) (top right). PC1, PC2 and PC3 represent the \% of explained variances. Technical replicates are shown with a red outline and overlap their replicate, therefore indicating the same gene expression. (B) Volcano plots of differential expression analysis (DEA). On the left is the Ct vs Cu comparison, on the right, the Pt vs Pu comparison. Red color indicates upregulated genes and blue downregulated genes in the treated samples (FDR $<0.05)$. Gray indicates non-differentially expressed genes (FDR $>0.05$ ). (C) Venn diagram intersection between the $\mathrm{Cb} 2$ effects in PCOS and control samples.

process; DNA/RNA regulation; Differentiation, proliferation and development; Circulatory system and development; Cell cycle; Carbohydrate metabolism; Biosynthetic process; Apoptosis; and Amino acid metabolism and protein folding, transport and localization (Fig. 2A). The GO terms associated to each category assigned by us is detailed in Supplementary Table 2, among which GO BP terms related to apoptosis were only enriched in PCOS cells (Fig. 2A).

Additionally, KEGG pathway analysis showed 29 pathways only dysregulated in PCOS LGCs treated and 17 pathways only dysregulated in non-PCOS treated.
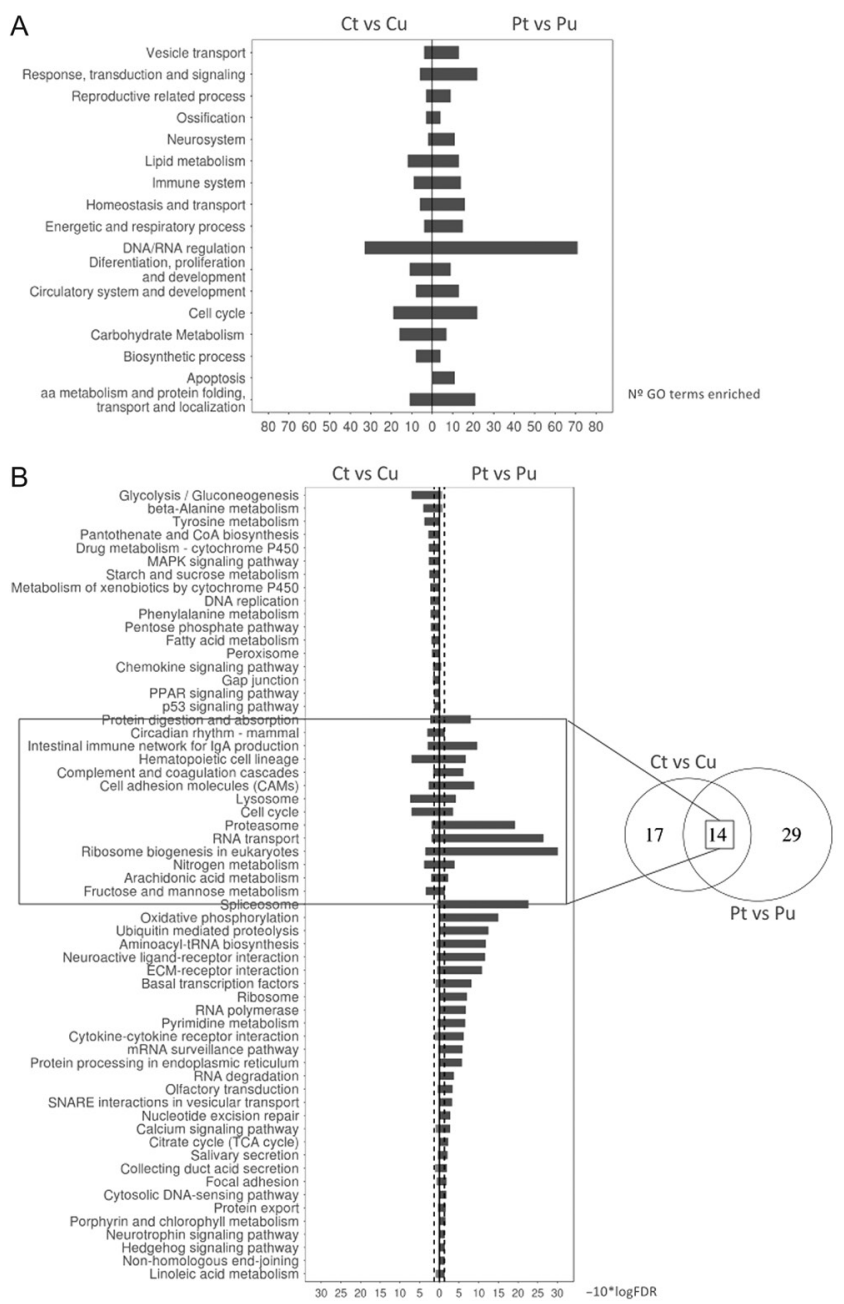

Figure 2 Functional enrichment of the $\mathrm{Cb} 2$ effects. (A) Gene Set Enrichment Analysis (GSEA) from Gene Ontology (GO). GO terms of the enriched biological processes $(\mathrm{BP})(\mathrm{FDR}<0.05)$ related to 17 general functions ( $Y$ axis) for the effects of $\mathrm{Cb} 2$ treatment on non-PCOS (Ct vs $\mathrm{Cu}$ ) and PCOS (Pt vs Pu) samples are indicated. The number of enriched GO BP terms is indicated on the $X$-axis. (B) GSEA according to Kyoto Encyclopedia of Genes and Genomes (KEGG) pathways for the effects of Cb2 on non-PCOS (Ct vs Cu) and PCOS (Pt vs Pu) LGCs. Significance is indicated on the $X$-axis (-log FDR). Only significant pathways are described on the $Y$-axis $(\mathrm{FDR}<0.05)$.

Interestingly, PCOs-treated LGCs shared 14 common Cb2-disrupted pathways with non-PCOS-treated LGCs. Protein digestion and absorption, the intestinal immune network for IgA production, the complement and coagulation cascade, cell adhesion molecules (CAM), the proteasome, RNA transport and ribosome biogenesis in eukaryotes were commonly dysregulated in Cb2-treated non-PCOS and PCOS LGCs but more highly enriched in the PCOS samples. Lysosomal and cell cycle pathways were also shared, but were more highly enriched in Cb2-treated non-PCOS (controls) LGCs (Fig. 2B). Metabolism of sugar, lipids and amino 
acids was also dysregulated after $\mathrm{Cb} 2$ treatment in both non-PCOS and PCOS cells.

Functional results from databases analyses (GO terms and KEGG pathways) showed more highly dysregulated pathways following $\mathrm{Cb} 2$ treatment in PCOS than in nonPCOS (control) LGCs (Fig. 2). In addition, the paired design removes changes related to the baseline and inter-patient variability, improving statistical power to detect differences and highlighting the treatment effect. KEGG pathways analysis in Pu vs $\mathrm{Cu}$ showed pathways that were dysregulated in $\mathrm{Pu} v \mathrm{Vs}$, but were not dysregulated in Pt vs Pu or Ct vs $\mathrm{Cu}$, indicating that the baseline differences in PCOS did not affect our results (Supplementary Fig. 1C).

\section{Functional network modeling between the main pathways altered in LGCs treated with $\mathrm{Cb2}$}

After the functional description of $\mathrm{Cb} 2$ treatment effect in PCOS vs controls, we highlighted the apoptosis function (hsa04210) because its enrichment was evident only in treated PCOS LGCs and because most of the enriched processes, such as cytokine-cytokine interactions, protein processing in the endoplasmic reticulum (ER), calcium signaling pathway, MAPK pathways, P53 signaling, lysosomes and the cell cycle, are directly involved in apoptotic responses (Supplementary Fig. 2). Moreover, these enriched processes (Fig. 2) are also directly involved in important pathways implicated in PCOS and Cb2 treatment, such as VEGF signaling (hsa04370), ovarian steroidogenesis (hsa04913) and circadian rhythm (hsa04710), which were previously described in the literature. For this reason, we focused our study on intersecting the mentioned pathways with the main pathways related to $\mathrm{Cb} 2$ response in the KEGG drug database (neuroactive ligand-receptor interactions (hsa04080) and the dopaminergic synapse (hsa04728)).
Finally, network analysis showed the principal genes that intersected between these pathways (Fig. 3). In this regard, we described genes involved in the selected pathways that might be key genes in Cb2 effect in PCOS, and they could help us to understand the underlying mechanism in PCOS. Among these key genes, AKT, $M A P K$ and $C R E B$ were of particular interest because they were the genes most shared between pathways.

\section{Validation of microarray data}

To validate whether there were significant differences between $\mathrm{Cb} 2$-treated and -untreated samples, seven key genes were selected by GSEA analysis, based not only on the significance of these genes, but also on their intersection with the selected pathway. These were validated by RT-qPCR from the same RNA samples used in the microarray. These genes could be grouped as biomarkers of the dopaminergic synapsis (MAOA), VEGF signaling (VEGF, AKT1, MAPK14) and apoptosis (CASP9, AIF). The circadian gene $B M A L 1$ was also selected and validated due to its importance in the activation of estrogen synthesis and aromatase expression.

The RT-qPCR results for all validated genes showed the same trend as found in the microarray experiment, confirming the microarray data. In addition, most of the changes were significant $(P$ value $<0.05$ ) (Fig. 4). RT-qPCR data showed that $\mathrm{Cb} 2$ treatment affected the apoptosis pathway by decreasing CASP9, which was significant in the non-PCOS samples ( $P$ value $<0.05)$, while increasing $A I F$, which was significant in the PCOS samples (Fig. 4A and D). In addition, Cb2 significantly decreased BMAL1 but increased MAOA in the PCOS samples, showing the same tendency as found in the microarray experiment (Fig. 4B and E). Finally, Cb2 treatment significantly decreased VEGFA in the non-PCOS and PCOS groups (non-PCOS $P$ value $<0.05$; PCOS $P$ value $<0.01$ ), while

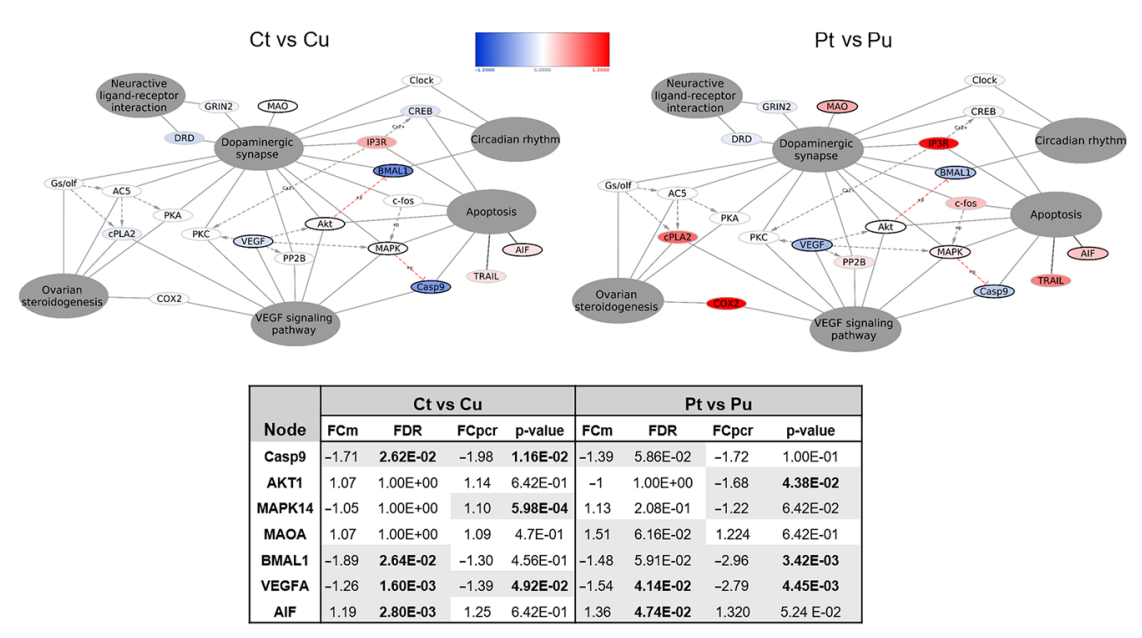

Figure 3 Network relationship between the main pathways and key intersected genes. Gene relationships between six pathways related to $\mathrm{PCOS}$ and the $\mathrm{Cb} 2$ drug response are indicated. On the top left, network expression values for non-PCOS treated with a D2-ag (Cb2) and on the top right, network expression values for PCOS treated with $\mathrm{Cb} 2$. The differences in color intensity of the genes indicate higher or lower fold changes (FC). Upregulation is shown in red and downregulation in blue. Table indicates fold changes in the microarray experiment (FCm), the false discovery rates (FDR) after the differential expression analysis (DEA), and the FC and $P$ values for real-time PCR (FCpcr). FDR $<0.1$ or $P$ value $<0.1$ is highlighted in gray. $\mathrm{FDR}<0.05$ or $P$ value $<0.05$ is in bold. 

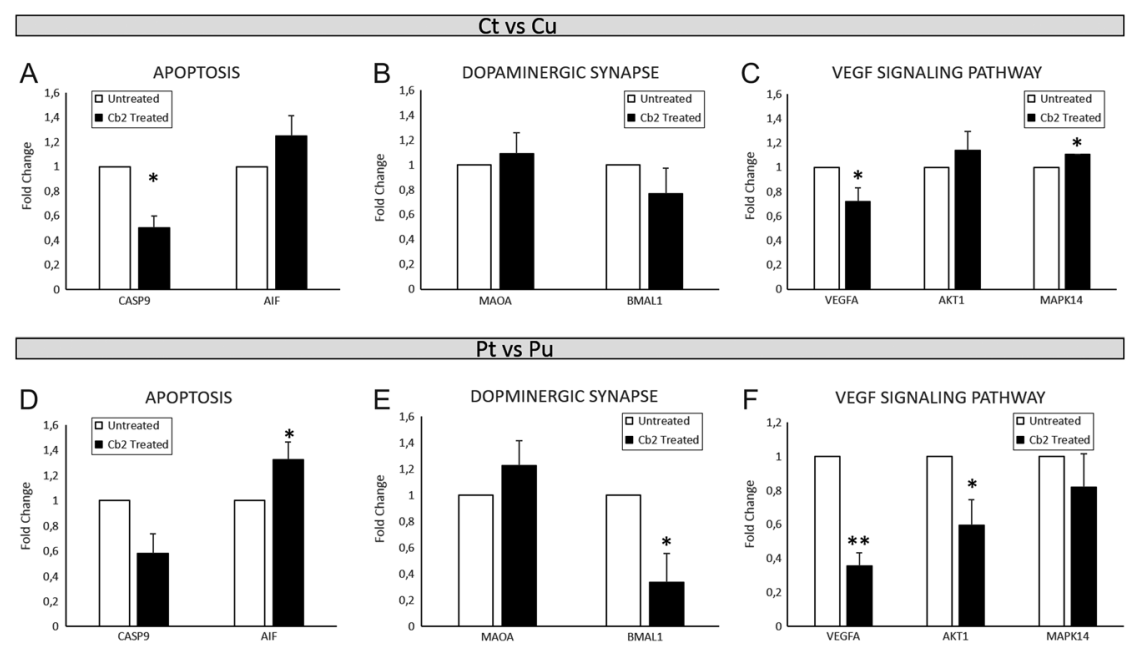

Figure 4 Validation of microarray data of genes up or downregulated in non-PCOS and PCOS LGCs treated with Cb2. Expression levels of genes related to the pathways: Apoptosis: (CASP9, AIF) in (A) non-PCOS and (D) PCOS; dopaminergic synapse (MAOA, BMAL1) in (B) non-PCOS and (E) PCOS and VEGF signaling pathway (VEGFA, AKT1, MAPK14) in (C) non-PCOS and (F) PCOS. Expression levels were determined by quantitative real-time PCR and normalized to the house keeping gene $A C T B$, quantified by the $\Delta \Delta C t$ method and expressed as the fold change in each group (mean \pm S.D.). Ct: non-PCOS (control) treated $(n=3)$; Cu: non-PCOS (control) untreated $(n=3)$; Pt: PCOS treated $(n=3)$; Pu: PCOS untreated $(n=3) . * P<0.05 ; * * P<0.01$ comparing $\mathrm{Cb}-2$ treated LGCs vs untreated group.
AKT1 and MAPK14 increased in the non-PCOS and decreased in the PCOS samples. The change in AKT1 was significant in PCOS cells ( $P$ value $<0.05$ ), and the change in MAPK 14 was significant in non-PCOS cells $(P$-value $<0.05)$ (Fig. 4C and F).

\section{Discussion}

The goal of this study was to understand the PCOS ovarian physiology from an 'omic' point of view, through exploring the mechanisms that underlie syndrome based on the therapeutic effects of $\mathrm{Cb} 2$. For this purpose, we used a transcriptomic and systems biology approach to better understand how $\mathrm{Cb} 2$ treatment influences ovarian function in PCOS in a holistic manner. One of important aspects of systems biology approaches is to identify the biological pathways or networks that connect the differing elements of a system. We have thus identified genes and their functional interactions in non-PCOS and PCOS LGCs treated with a D2-ag (Cb2) to provide novel information about the mechanisms associated with $\mathrm{Cb} 2$ treatment and its relationship in both physiological and pathological conditions.

Because genes are not independent variables, Gene Set Enrichment Analysis (GSEA) better mirrors the biology underlying the systems we seek to study than artificial thresholds as fold changes and/or adj- $P$ values (Subramanian et al. 2005, Dalman et al. 2012, Khatri et al. 2012). GSEA using GO and KEGG databases showed common and specific dysregulated processes in non-PCOS (control) and PCOS LGCs treated with Cb2. It is important to note that, although some functions were more highly affected in non-PCOS samples, the $\mathrm{Cb} 2$-affected biological functions and pathways were generally more significantly altered in PCOS LGCs, suggesting that the $\mathrm{Cb} 2$ treatment response was more robust for the PCOS samples. In this regard, our functional enrichment study provides novel information about processes affected by $\mathrm{Cb} 2$ treatment. In addition, we wanted to better understand the enhanced effect of $\mathrm{Cb} 2$ on PCOS cells and highlight new key genes using other systems biology approaches, such as network intersection between pathways that exploits pathway knowledge in public repositories such as GO or KEGG, rather than on methods that infer pathways from molecular measurements (Khatri et al. 2012). For this purpose, we intersected pathways implicated in PCOS and/or Cb2 treatment associated with the enriched processes we identify, and which have been previously described in the literature, such as VEGF signaling, ovarian steroidogenesis, circadian rhythm and apoptosis. These pathways intersected with the main pathways related to $\mathrm{Cb} 2$ response in the KEGG drug database, such as neuroactive ligand-receptor interactions and the dopaminergic synapse.

Follicular development is associated with intense angiogenesis and increased vascular permeability under the control of angiogenic factors, such as VEGFA, which is essential for corpus luteum angiogenesis (Ferrara 1995), increases proliferation and inhibits apoptosis of GCs (Irusta et al. 2010). Previous studies from our group demonstrated a D2-ag dose-dependent inhibition in VEGF production and secretion in LGCs (Ferrero et al. 2014a,b) at a post-transcriptional rather than transcriptional level (Ferrero et. al. 2015), suggesting that efficacy of D2-ags in preventing OHSS might rely on their capacity to inhibit VEGF protein secretion by LGCs. However, this study demonstrated that VEGFA mRNA levels were significantly decreased in nonPCOS and PCOS LGCs treated with Cb2. One possible explanation for not finding mRNA-level differences in our previous study might be attributable to the derivation of LGCs from non-PCOS and PCOS patients treated with or without $\mathrm{Cb} 2$ from separate women; in contrast, this new study is a paired design that compared for the first time untreated vs treated LGCs from the same patient. In 
this way, it avoids inter-patient variability and improves statistical power to detect differences, making this new study more sensitive and accurate.

Of note, despite the fact that women with PCOS overexpress and oversecrete VEGF during the luteal phase after the induction of ovulation (Neulen et al. 1995, Agrawal et al. 2002), we found that Cb2 efficiently inhibits VEGF in both PCOS and non-PCOS LGCs, and thereby could prevent OHSS.

Functions related to apoptosis, including cytokinecytokine interactions, protein processing in the endoplasmic reticulum (ER) and calcium signaling, were especially dysregulated in our GSEA in PCOS, while MAPK pathways and P53 signaling were in non-PCOS. Lysosomes and the cell cycle were dysregulated in both (Supplementary Fig. 2). Our results confirmed findings in previous studies, which showed that dopamine induces apoptosis in PCOS-derived GCs (Saller et al. 2014). However, our mRNA expression data showed a significant decrease in CASP9, which plays an important role in apoptosis. Recently, a previous study reported that AIF might serve as a key factor during follicular atresia in GCs associated with ER stress (Yang et al. 2017). In this regard, we observed a significant increase in AIF in both LGCs from women without PCOS and women with PCOS treated with $\mathrm{Cb} 2$, suggesting for the first time that AIF could be regulating the apoptosis of PCOS GCs treated with Cb2 through ER stress. This caspase-independent apoptotic sub-pathway could be an alternative pathway that is not described in KEGG and is involved in the ovarian PCOS physiology. Thus, we bring new insight for future studies to analyze how $\mathrm{Cb} 2$ regulates apoptosis.

Microarray data showed that AKT and MAPK14 were increased in non-PCOS and decreased in PCOS LGCs treated with $\mathrm{Cb} 2$. Several studies have described the important role of AKT and MAPK14 in LGCs during folliculogenesis, due to their involvement in cellular processes including cell growth, survival, proliferation, metabolism and apoptosis (Villa-Diaz \& Miyano 2004, Cecconi et al. 2010). Our systems biology results corroborate and reinforce the main role of these genes, which intersect directly with the dopaminergic synapse, apoptosis, VEGF signaling and circadian rhythm pathways. New studies will be necessary to explain why $\mathrm{Cb} 2$ treatment has differing effects on non-PCOS and PCOS cells.

Women with PCOS present increased MAO activity in hyperluteinized ovaries and GCs, which might be attributed to the increased number of transformed corpus luteum as a consequence of diminished luteolysis (IvaniÅeviÄ-MilovanoviÄ et al. 2003, Saller et al. 2014). This increased MAO-A/B activity might be associated with the increase in dopamine metabolism in GCs derived from women with PCOS previously described by our group (Gómez et al. 2011). Here, we observed an increase in MAOA expression levels in non-PCOS and in LGCs from PCOS patients treated with $\mathrm{Cb} 2$, which was significant in the PCOS samples, suggesting increased metabolism of dopamine in $\mathrm{Cb}-2$ treated derived from PCOS patients.

Finally, our transcriptomic data from LGCs treated with $\mathrm{Cb} 2$ showed that BMAL1, a circadian gene involved in activation of estrogen synthesis and aromatase expression, was decreased in both PCOS and nonPCOS samples treated with Cb2. Hyperandrogenism is implicated in the genesis of PCOS and affects the expression of circadian genes, such as BMAL1. PCOS LGCs exhibit hyperandrogenism (Eisner et al. 2002), low BMAL1 expression (Zhang et al. 2016) and low follicular estrogen levels, which cause antral follicle arrest and anovulation. In this regard, our results suggest that $\mathrm{Cb} 2$ treatment in PCOS further decreases BMAL1, increasing the hyperandrogenism risk in these patients. Future studies will be necessary to analyze whether Cb2 has side effects during PCOS treatment related to genes involved in ovarian steroidogenesis, such as cyclooxygenase 2 (COX2) and cytosolic phospholipase A2 CPLA2, and decreasing BMAL1 that is involved in estrogen synthesis.

This is a preliminary descriptive in vitro study with a limited number of samples. However, we have designed a paired study and validated genes by two mRNA quantification techniques that reinforce our findings.

In summary, LGCs from PCOS cells treated with Cb2 present more significant changes at the transcriptomic level than non-PCOS cells. The network analysis between the main pathways highlighted CASP9, VEGFA, $A K T 1, C R E B, A I F, M A O A, M A P K 14$ and BMAL1 as key genes implicated in the $\mathrm{Cb} 2$ response and in the molecular mechanisms of PCOS.

\section{Supplementary data}

This is linked to the online version of the paper at https://doi.org/10.1530/REP-18-0027.

\section{Declaration of interest}

The authors declare that there is no conflict of interest that could be perceived as prejudicing the impartiality of the research reported.

\section{Funding}

This work was supported by the Spanish Ministry of Economy and Competitiveness through the Miguel Servet Program (CP13/00077) cofounded by the FEDER (European Regional Development Fund), the Carlos III Institute of Health grant (PI14/00547) awarded to R G, Valencian State Governments through GV/2016/032 awarded to H F and supported by the Spanish Ministry of Economy and Competitiviness through the Sara Borrell Program (CD15/00057) awarded to H F. Carlos III Institute of Health grant (PI15/00312) awarded to A P. 


\section{Acknowledgements}

The authors express their sincere thanks to the participants of this study who made this work possible. The authors also offer special thanks to all the medical and technical staff of the IVI Clinic for their assistance and contribution in obtaining samples of the follicular fluid.

\section{References}

Agrawal R, Jacobs H, Payne N \& Conway G 2002 Concentration of vascular endothelial growth factor released by cultured human luteinized granulosa cells is higher in women with polycystic ovaries than in women with normal ovaries. Fertility and Sterility $\mathbf{7 8}$ 1164-1169. (https://doi.org/10.1016/S0015-0282(02)04242-5)

Al-Edani T, Assou S, Ferrières A, Bringer Deutsch S, Gala A, Lecellier CH, Ait-Ahmed O \& Hamamah S 2014 Female aging alters expression of human cumulus cells genes that are essential for oocyte quality. BioMed Research International 2014 964614. (https://doi. org/10.1155/2014/964614)

Ashburner M, Ball CA, Blake JA, Botstein D, Butler H, Cherry JM, Davis AP, Dolinski K, Dwight SS, Eppig JT et al 2000 Gene ontology: tool for the unification of biology. Nature Genetics 25 25-29. (https:// doi.org/10.1038/75556)

Barabási A-L, Gulbahce N \& Loscalzo J 2011 Network medicine: a network-based approach to human disease. Nature Reviews Genetics 12 56-68. (https://doi.org/10.1038/nrg2918)

Benjamini Y \& Hochberg Y 1995 Controlling the false discovery rate: a practical and powerful approach to multiple testing. Journal of the Royal Statistical Society: Series B 57 289-300.

Bolstad BM, Irizarry RA, Åstrand M \& Speed TP 2003 A comparison of normalization methods for high density oligonucleotide array data based on variance and bias. Bioinformatics 19 185-193. (https://doi. org/10.1093/bioinformatics/19.2.185)

Cecconi S, Rossi G, Santilli A, Di Stefano L, Hoshino Y, Sato E, Palmerini MG \& Macchiarelli G 2010 Akt expression in mouse oocytes matured in vivo and in vitro. Reproductive BioMedicine Online 20 35-41. (https://doi.org/10.1016/j.rbmo.2009.10.011)

Cline MS, Smoot M, Cerami E, Kuchinsky A, Landys N, Workman C, Christmas R, Avila-Campilo I, Creech M, Gross B et al. 2007 Integration of biological networks and gene expression data using Cytoscape. Nature Protocols 2 2366-2382. (https://doi.org/10.1038/nprot.2007.324)

Dalman MR, Deeter A, Nimishakavi G \& Duan Z-H 2012 Fold change and $\mathrm{p}$-value cutoffs significantly alter microarray interpretations. BMC Bioinformatics 13 S2-S11. (https://doi.org/10.1186/1471-2105-13S2-S11)

Douville G \& Sirard M-A 2014 Changes in granulosa cells gene expression associated with growth, plateau and atretic phases in medium bovine follicles. Journal of Ovarian Research 7 50. (https://doi.org/10.1186/17572215-7-50)

Eisner JR, Barnett MA, Dumesic DA \& Abbott DH 2002 Ovarian hyperandrogenism in adult female rhesus monkeys exposed to prenatal androgen excess. Fertility and Sterility 77 167-172. (https://doi. org/10.1016/S0015-0282(01)02947-8)

Ferrara N 1995 The role of vascular endothelial growth factor in pathological angiogenesis. Breast Cancer Research and Treatment $\mathbf{3 6}$ 127-137. (https://doi.org/10.1007/BF00666035)

Ferrero H, Delgado-Rosas F, Garcia-Pascual CM, Monterde M, Zimmermann RC, Simón C, Pellicer A \& Gómez R 2012 Efficiency and purity provided by the existing methods for the isolation of luteinized granulosa cells: a comparative study. Human Reproduction 27 1781-1789. (https://doi.org/10.1093/humrep/des096)

Ferrero H, Garcia-Pascual CM, Gaytán M, Morales C, Simón C, Gaytán F, Pellicer A \& Gómez R 2014a Dopamine receptor 2 activation inhibits ovarian vascular endothelial growth factor secretion in an ovarian hyperstimulation syndrome (OHSS) animal model: implications for treatment of OHSS with dopamine receptor 2 agonists. Fertility and Sterility 102 1468-1476. (https://doi.org/10.1016/j.fertnstert.2014.07.1240)
Ferrero H, Garcia-Pascual CM, Gómez R, Delgado-Rosas F, Cauli O, Simón C, Gaytán F \& Pellicer A 2014b Dopamine receptor 2 activation inhibits ovarian vascular endothelial growth factor secretion in vitro: implications for treatment of ovarian hyperstimulation syndrome with dopamine receptor 2 agonists. Fertility and Sterility 101 1411-1418. (https://doi.org/10.1016/j.fertnstert.2014.01.031)

Ferrero H, García-Pascual CM, Pellicer N, Simón C, Pellicer A \& Gómez R 2015 Dopamine agonist inhibits vascular endothelial growth factor protein production and secretion in granulosa cells. Reproductive Biology and Endocrinology 13 104. (https://doi.org/10.1186/s12958015-0102-4)

Golini VE, Stradaioli G \& Sirard MA 2014 Transcriptome analysis of bovine granulosa cells of preovulatory follicles harvested 30, 60, 90, and 120 days postpartum. Theriogenology 82 580-591. (https://doi. org/10.1016/j.theriogenology.2014.05.019)

Gómez R, Ferrero H, Delgado-Rosas F, Gaytan M, Morales C, Zimmermann RC, Simón C, Gaytan F \& Pellicer A 2011 Evidences for the existence of a low dopaminergic tone in polycystic ovarian syndrome: implications for OHSS development and treatment. Journal of Clinical Endocrinology and Metabolism 96 2484-2492. (https://doi. org/10.1210/jc.2011-0075)

Goverde AJ, Van Koert AJB, Eijkemans MJ, Knauff EAH, Westerveld HE, Fauser B \& Broekmans FJ 2009 Indicators for metabolic disturbances in anovulatory women with polycystic ovary syndrome diagnosed according to the Rotterdam consensus criteria. Human Reproduction 24 710-717. (https://doi.org/10.1093/humrep/den433)

Guvendag Guven ES, Dilbaz S, Duraker R, Mentese A, Cinar O \& Ozdegirmenci O 2013 The effect of cabergoline on folicular microenviroment profile in patients with high risk of OHSS. Gynecological Endocrinology 29 749-753. (https://doi.org/10.3109/09 513590.2013.801440)

Hsueh AJ \& Rauch R 2012 Ovarian kaleidoscope database: ten years and beyond. Biology of Reproduction 86 192. (https://doi.org/10.1095/ biolreprod.112.099127)

Irizarry RA, Hobbs B, Collin F, Beazer-Barclay YD, Antonellis KJ, Scherf U \& Speed TP 2003 Exploration, normalization, and summaries of high density oligonucleotide array probe level data. Biostatistics 4 249-264. (https://doi.org/10.1093/biostatistics/4.2.249)

Irusta G, Abramovich D, Parborell F \& Tesone M 2010 Direct survival role of vascular endothelial growth factor (VEGF) on rat ovarian follicular cells. Molecular and Cellular Endocrinology 325 93-100. (https://doi. org/10.1016/j.mce.2010.04.018)

IvaniÅeviÄ-MilovanoviÄ OK, Demajo M, PetroviÄ V \& Cvijï̈ G 2003 Total monoamine oxidase activity in the hypothalamus, ovary and uterus of rats with an extreme number of ovarian corpora lutea. Acta Physiologica Hungarica 90 319-325. (https;//doi.org/10.1556/APhysiol.90.2003.4.5)

Kanehisa M, Goto S, Sato Y, Furumichi M \& Tanabe M 2012 KEGG for integration and interpretation of large-scale molecular data sets. Nucleic Acids Research 40 D109-D114. (https://doi.org/10.1093/nar/gkr988)

Karuputhula NB, Chattopadhyay R, Chakravarty B \& Chaudhury K 2013 Oxidative status in granulosa cells of infertile women undergoing IVF. Systems Biology in Reproductive Medicine 59 91-98. (https://doi.org/10 .3109/19396368.2012.743197)

Khatri P, Sirota M \& Butte AJ 2012 Ten years of pathway analysis: current approaches and outstanding challenges. PLOS Computational Biology $\mathbf{8}$ e1002375. (https://doi.org/10.1371/journal.pcbi.1002375)

Lan C-W, Chen M-J, Tai K-Y, Danny CW, Yang Y-C, Jan P-S, Yang Y-S, Chen H-F \& Ho H-N 2015 Functional microarray analysis of differentially expressed genes in granulosa cells from women with polycystic ovary syndrome related to MAPK/ERK signaling. Scientific Reports 514994. (https://doi.org/10.1038/srep14994)

Mary MJ, Vetrivel U, Munuswamy D \& Melanathuru V 2016 PCOSDB: PolyCystic Ovary Syndrome Database for manually curated disease associated genes. Bioinformation 12 4. (https://doi. org/10.6026/97320630012004)

Montaner D \& Dopazo J 2010 Multidimensional gene set analysis of genomic data. PLOS ONE 5 e10348. (https://doi.org/10.1371/journal. pone.0010348)

Neulen J, Yan Z, Raczek S, Weindel K, Keck C, Weich HA, Marme D \& Breckwoldt M 1995 Human chorionic gonadotropin-dependent 
expression of vascular endothelial growth factor/vascular permeability factor in human granulosa cells: importance in ovarian hyperstimulation syndrome. Journal of Clinical Endocrinology and Metabolism 80 1967-1971. (https://doi.org/10.1210/jcem.80.6.7775647)

Norman RJ \& Clark AM 1998 Obesity and reproductive disorders: a review. Reproduction, Fertility and Development 10 55-63. (https://doi. org/10.1071/R98010)

Ouandaogo ZG, Haouzi D, Assou S, Dechaud H, Kadoch IJ, De Vos J \& Hamamah S 2011 Human cumulus cells molecular signature in relation to oocyte nuclear maturity stage. PLOS ONE 6 e27179. (https://doi. org/10.1371/journal.pone.0027179)

Papaleo E, Doldi N, De Santis L, Marelli G, Marsiglio E, Rofena S \& Ferrari A 2001 Cabergoline influences ovarian stimulation in hyperprolactinaemic patients with polycystic ovary syndrome. Human Reproduction 16 2263-2266. (https://doi.org/10.1093/humrep/16.11.2263)

Pasquali R \& Gambineri A 2006 Polycystic ovary syndrome: a multifaceted disease from adolescence to adult age. Annals of the New York Academy of Sciences 1092 158-174. (https://doi.org/10.1196/annals.1365.014)

R Core Team \& R Foundation for Statistical Computing 2013 R: A Language and Environment for Statistical Computing. ISBN 3-900051-07-0, URL: http://www.R-project.org.

Ritchie ME, Phipson B, Wu D, Hu Y, Law CW, Shi W \& Smyth GK 2015 Limma powers differential expression analyses for RNA-sequencing and microarray studies. Nucleic Acids Research 43 e47. (https://doi. org/10.1093/nar/gkv007)

Saller S, Kunz L, Berg D, Berg U, Lara H, Urra J, Hecht S, Pavlik R, Thaler CJ \& Mayerhofer A 2014 Dopamine in human follicular fluid is associated with cellular uptake and metabolism-dependent generation of reactive oxygen species in granulosa cells: implications for physiology and pathology. Human Reproduction 29 555-567. (https://doi.org/10.1093/ humrep/det422)

Sartor MA, Leikauf GD \& Medvedovic M 2009 LRpath: a logistic regression approach for identifying enriched biological groups in gene expression data. Bioinformatics 25 211-217. (https://doi.org/10.1093/ bioinformatics/btn592)
Subramanian A, Tamayo P, Mootha VK, Mukherjee S, Ebert BL, Gillette MA, Paulovich A, Pomeroy SL, Golub TR, Lander ES \& Mesirov JP 2005 Gene set enrichment analysis: a knowledge-based approach for interpreting genome-wide expression profiles. PNAS 102 15545-15550. (https://doi. org/10.1073/pnas.0506580102)

Swanton A, Storey L, McVeigh E \& Child T 2010 IVF outcome in women with PCOS, PCO and normal ovarian morphology. European Journal of Obstetrics and Gynecology and Reproductive Biology 149 68-71. (https://doi.org/10.1016/j.ejogrb.2009.11.017)

Van Wely M, Bayram N \& der Veen F 2003 Recombinant FSH in alternative doses or versus urinary gonadotrophins for ovulation induction in subfertility associated with polycystic ovary syndrome: a systematic review based on a Cochrane review. Human Reproduction 18 1143-1149. (https://doi.org/10.1093/humrep/deg229)

Villa-Diaz LG \& Miyano T 2004 Activation of p38 MAPK during porcine oocyte maturation. Biology of Reproduction 71 691-696. (https://doi. org/10.1095/biolreprod.103.026310)

Yang D, Jiang T, Lin P, Chen H, Wang L, Wang N, Zhao F, Wang A \& Jin Y 2017 Knock-down of apoptosis inducing factor gene protects endoplasmic reticulum stress-mediated goat granulosa cell apoptosis. Theriogenology 88 89-97. (https://doi.org/10.1016/j.theriogenology.2016.10.001)

Zhang J, Liu J, Zhu K, Hong Y, Sun Y, Zhao X, Du Y \& Chen Z-J 2016 Effects of BMAL1 - SIRT1-positive cycle on estrogen synthesis in human ovarian granulosa cells: an implicative role of BMAL1 in PCOS. Endocrine $\mathbf{5 3}$ 574-584. (https://doi.org/10.1007/s12020-016-0961-2)

Received 16 January 2018

First decision 26 January 2018

Revised manuscript received 2 February 2018

Accepted 9 February 2018 